\title{
Limits of sympathetic cooling of fermions: The role of the heat capacity of the coolant
}

\author{
L. D. Carr* and Y. Castin \\ Laboratoire Kastler Brossel, Ecole Normale Supérieure, \\ 24 rue Lhomond, 75231 Paris CEDEX 05, France
}

(Dated: November 5, 2018)

\begin{abstract}
The sympathetic cooling of an initially degenerate Fermi gas by either an ideal Bose gas below $T_{c}$ or an ideal Boltzmann gas is investigated. It is shown that the efficiency of cooling by a Bose gas below $T_{c}$ is by no means reduced when its heat capacity becomes much less than that of the Fermi gas, where efficiency is measured by the decrease in the temperature of the Fermi gas per number of particles evaporated from the coolant. This contradicts the intuitive idea that an efficient coolant must have a large heat capacity. In contrast, for a Boltzmann gas a minimal value of the ratio of the heat capacities is indeed necessary to achieve $T=0$ and all of the particles must be evaporated.
\end{abstract}

PACS numbers:

\section{INTRODUCTION}

Sympathetic cooling of fermionic atoms has proven to be an efficient tool to produce strongly degenerate Fermi gases [1, 2, 3, 4, 5]. It is often thought that when the specific heat of the coolant becomes less than that of the fermions the cooling process slows drastically and even comes to a halt [1, 6, 7]. This belief derives from the following argument based on standard thermodynamics [8]. A coolant is not only described by its temperature but also by its heat capacity. The equilibrium temperature between two bodies initially at different temperatures $T_{f}$ and $T_{b}$ is $T=\left(C_{f} T_{f}+C_{b} T_{b}\right) /\left(C_{f}+C_{b}\right)$, where $C_{f}$ and $C_{b}$ are the heat capacities of the two bodies [9]. Therefore, regardless of having a very cold object $b\left(T_{b} \ll T_{f}\right)$, it will not act as a good coolant unless it is also true that $C_{b} \gg C_{f}$.

However, this argument does not include the evaporation of atoms from the coolant 10, 11], a key ingredient of sympathetic cooling. In the following, we shall consider the ideal case of sympathetic cooling, where all losses of particles due to inelastic collisions are absent. One can then evaporate sufficiently slowly that the Fermi gas and the coolant remain close to thermal equilibrium. This is in the spirit of the above argument, based on heat capacities, as heat capacity is a property of an equilibrium system. In this case, the only limitation of resources is the number of particles in the coolant. We then introduce an idealized model of sympathetic cooling: the atomic motion is treated in the semi-classical approximation and cooling proceeds in discrete steps. In each step, the atoms of the coolant go through an evaporation phase, and then the fermions and the coolant thermalize (see Fig. 1 below). This is a generalization of the discrete scheme used for evaporative cooling in Ref. 12], to the case of sympathetic cooling. A similar semi-classical and discrete model for sympathetic cooling was put forward in Ref. [13], with the addition of features like collisional losses and space dependent effects in the evaporation process, but without a determination of the limits of the cooling process. Using our idealized model, we shall

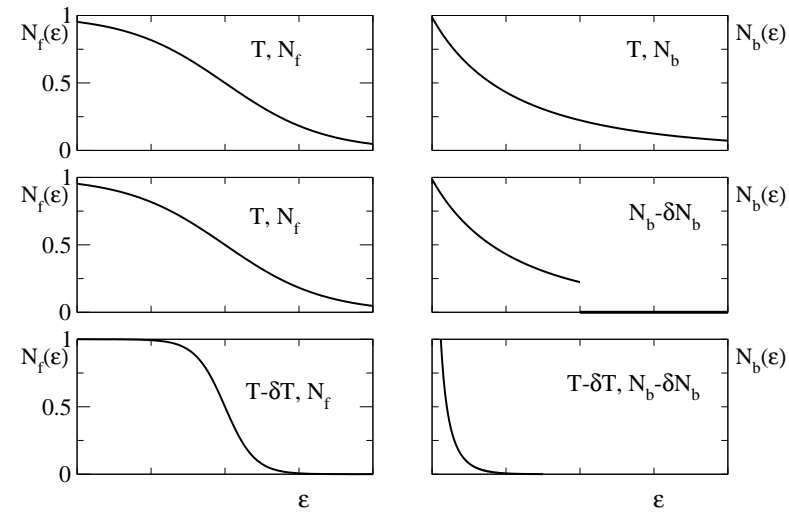

FIG. 1: Shown is a diagram of the evaporative cooling process in our model. In the upper two panels, a gas of $N_{f}$ fermions at temperature $T$ is in thermal equilibrium with a coolant of $N_{b}$ bosons or Boltzmann particles. In the middle two panels, the tail of the number distribution of the coolant is cut and $\delta N_{b}$ particles are removed. In the lower two panels, the systems are permitted to rethermalize, resulting in a reduced common temperature $T-\delta T$. The values of $\delta T$ and $\delta N_{b}$ have been exaggerated for the purposes of illustration; the units are arbitrary.

then show that the argument based on the heat capacities is approximately correct for fermions cooled by an ideal Boltzmann gas [14], but incorrect when cooled by an ideal Bose gas below the Bose condensation temperature $T_{c}$. In fact, in the latter case, it is preferential to have $C_{b} \ll C_{f}$ to minimize the number of particles of the coolant whose evaporation is required to reach zero temperature [15].

\section{THE MODEL}

We assume that the Fermi gas, denoted by the subscript $f$, and the coolant, denoted by the subscript $b$, are initially in thermal equilibrium at a common tempera- 
ture $T$. One evaporates all particles of the coolant with an energy greater than $\eta k_{B} T[10,11,12,[16]$, where $\eta$ is a constant, typically 5 to 7 in current experiments. This has the effect of removing a number of particles $\delta N_{b}$ and an energy $\delta E_{b}$ from the coolant:

$$
\begin{aligned}
\delta N_{b} & =\int_{\eta k_{B} T}^{\infty} d \epsilon \rho_{b}(\epsilon) N_{b}(\epsilon), \\
\delta E_{b} & =\int_{\eta k_{B} T}^{\infty} d \epsilon \rho_{b}(\epsilon) N_{b}(\epsilon) \epsilon,
\end{aligned}
$$

where $\rho_{b}(\epsilon)$ is the density of states of a particle in $b$ and $N_{b}(\epsilon)$ is the mean occupation number of a state of energy $\epsilon$. In what follows we restrict ourselves to the semiclassical regime in which the number of trap levels with an energy less than $k_{B} T$ is much larger than unity, both for species $f$ and $b$. Then the case of a density of states of the form

$$
\rho_{f, b}(\epsilon)=A_{f, b} \epsilon^{\delta+1 / 2}
$$

may be considered: this encompasses both a harmonic potential $(\delta=3 / 2)$ and a box $(\delta=0)$ [17]. Here the subscript $f(b)$ stands for the fermions (coolant). In the two limiting cases of a non-degenerate gas $N_{b}(\epsilon)=$ $\exp [-\beta(\epsilon-\mu)]$ and a Bose gas below $T_{c}, N_{b}(\epsilon)=$ $1 /[\exp (\beta \epsilon)-1]$, the mean energy of an evaporated particle [16] is independent of the chemical potential $\mu$ and proportional to $T$ :

$$
\frac{\delta E_{b}}{\delta N_{b}}=f(\eta) k_{B} T .
$$

The function $f(\eta)$ is different for a Bose gas below $T_{c}$ and for a Boltzmann gas, but has the same large $\eta$ behaviour in both cases [18]:

$$
f(\eta)=\eta+1+\frac{\delta+\frac{1}{2}}{\eta}+\mathcal{O}\left(\frac{1}{\eta^{2}}\right) .
$$

Once the particles are removed, the system is left to rethermalize. The decrease in temperature $\delta T$ is obtained by conservation of energy,

$$
\begin{array}{r}
E_{f}\left(N_{f}, T\right)+E_{b}\left(N_{b}, T\right)-\delta E_{b} \\
=E_{f}\left(N_{f}, T-\delta T\right)+E_{b}\left(N_{b}-\delta N_{b}, T-\delta T\right) .
\end{array}
$$

Note that $E_{f}$ is the total energy of the fermions, not the Fermi energy. The parameter $\eta$ is chosen sufficiently large so that $\delta N_{b} \ll N_{b}$ and $\delta T \ll T$. Equation (6) may be expanded to leading order in $\delta N_{b}$ and $\delta T$ :

$$
\frac{\delta T}{\delta N_{b}}=\frac{f(\eta) k_{B} T-\frac{\partial E_{b}}{\partial N_{b}}\left(N_{b}, T\right)}{C_{f}\left(N_{f}, T\right)+C_{b}\left(N_{b}, T\right)}
$$

where the heat capacities are defined by $C_{f} \equiv \partial_{T} E_{f}$ and $C_{b}=\partial_{T} E_{b}$.

Equation (7), valid for a Boltzmann gas or a degenerate Bose gas, will be studied for both a three dimensional

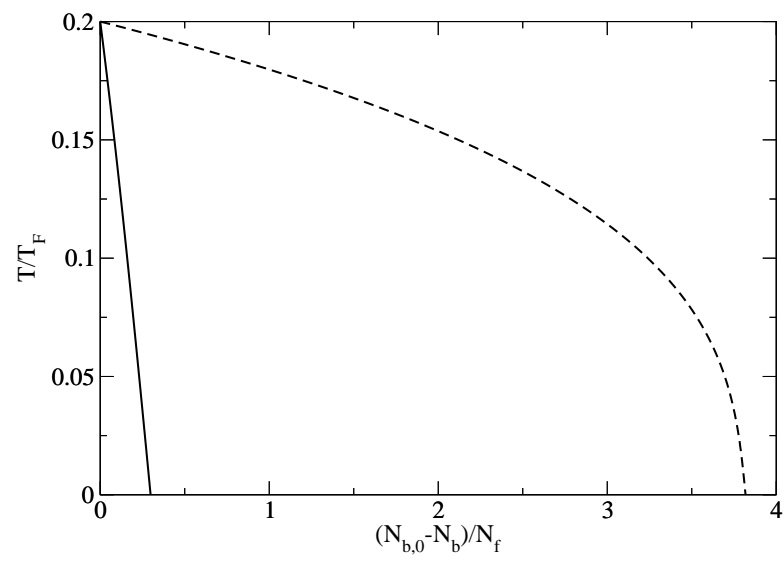

FIG. 2: Shown is the temperature of a degenerate Fermi gas sympathetically cooled by a Bose gas with a condensate present, as a function of the number of bosons evaporated in a harmonic trap, that is of the difference between the initial number of bosons $N_{b, 0}$ (assumed to fulfill Eq. (14) ) and the final number $N_{b}$. Two cases are depicted: $\omega_{f} / \omega_{b}=1$ (solid line) and $\omega_{f} / \omega_{b}=5$ (dashed line) where $\omega_{f} / \omega_{b}$ is the ratio of the mean trapping frequencies of the fermions and bosons. It is apparent that $\omega_{f} / \omega_{b} \lesssim 1$ requires a greatly reduced number of bosons in order to cool to $T=0$, and may therefore be considered as a more efficient experimental configuration. Here the choice of parameters was $\eta=6$ for the energy cut-off and $T_{0} / T_{F}=0.2$, where $T_{0}$ is the initial temperature.

box and a harmonic potential. In the former case, the mean field energy per particle of the coolant, due to the interaction with the Fermi gas, is constant and therefore plays no role. In the latter case, it is not exactly constant, but we shall assume that it is negligible as compared to $k_{B} T$. Additionally, for simplicity it will be assumed that the initial state of the fermions is degenerate. In this case the total energy is

$$
\begin{array}{r}
E_{f}\left(N_{f}, T\right)=E_{f}\left(N_{f}, 0\right) \\
+\alpha N_{f} k_{B} \frac{T^{2}}{T_{F}}\left\{1+\kappa\left(\frac{T}{T_{F}}\right)^{2}+\mathcal{O}\left[\left(\frac{T}{T_{F}}\right)^{4}\right]\right\},
\end{array}
$$

where $\alpha \equiv \pi^{2}(\delta+3 / 2) / 6, \kappa \equiv \pi^{2}(\delta+1 / 2)(\delta-3) / 20$, $\delta$ is the exponent in the density of states and the Fermi temperature $T_{F}$ is given by

$$
A_{f}\left(k_{B} T_{F}\right)^{\delta+3 / 2}=(\delta+3 / 2) N_{f} .
$$

In Eq. (8) we shall keep terms only to second order in $T$ in the following.

\section{COOLING BY AN IDEAL BOSE GAS BELOW $T_{c}$}

A non-interacting Bose gas with a temperature below $T_{c}$ has a total energy

$$
E_{b}\left(N_{b}, T\right)=\frac{\nu A_{b}}{\delta+5 / 2}\left(k_{B} T\right)^{\delta+5 / 2}
$$


where $\nu \equiv(\delta+5 / 2) \Gamma(\delta+5 / 2) \zeta(\delta+5 / 2)$ is a constant and $\Gamma$ and $\zeta$ are gamma and zeta functions, respectively 19$]$. Note that $E_{b}$ does not depend on the total number of bosons, since the occupation of the excited states is saturated to its maximal value in the presence of a condensate. Therefore $\partial E_{b} / \partial N_{b}=0$ and Eq. (17) reduces to

$$
\frac{\delta T}{\delta N_{b}}=\frac{f(\eta) T_{F}}{2 \alpha N_{f}\left[1+\frac{3 \nu}{\pi^{2}} \frac{A_{b}}{A_{f}}\left(\frac{T}{T_{F}}\right)^{\delta+1 / 2}\right]}
$$

where we used the fact that $A_{f}$ is related to the Fermi temperature $T_{F}$ by Eq. (9). When species $f$ and $b$ are in a box, $A_{b} / A_{f}$ is simply the ratio $V_{b} / V_{f}$ of the spatial volumes occupied by the two species. When species $f$ and $b$ are trapped harmonically, $A_{b} / A_{f}$ is given by the ratio $\left(\omega_{f} / \omega_{b}\right)^{3}$ where $\omega_{f, b}$ are the geometric means of the three oscillation frequencies for species $f$ and $b$, respectively.

The term in $T^{\delta+1 / 2}$ in the denominator of Eq. (11) represents the ratio of the two heat capacities:

$$
\frac{C_{b}}{C_{f}}=\frac{3 \nu}{\pi^{2}} \frac{A_{b}}{A_{f}}\left(\frac{T}{T_{F}}\right)^{\delta+1 / 2}
$$

If one measures the efficiency of the cooling by the temperature decrease per number of bosons evaporated, i.e., $\delta T / \delta N_{b}$, it is most efficient to have $C_{b} \ll C_{f}$. In this case the first term in the denominator dominates and the derivative is maximized. In Fig. 2 is shown the temperature as a function of the number of bosons evaporated, as can be obtained from the solution of the differential equation Eq. (11) for the case of harmonic trapping and $\eta=6$. It is apparent that it is advantageous to evaporative cooling to choose a trap frequency ratio $\omega_{f} / \omega_{b} \lesssim 1$, in order to maintain $C_{b} / C_{f}$ small at typical experimental temperatures $T / T_{F} \sim 0.2$ or 0.1 .

A simple condition may be obtained from Eq. (11) for the total initial number of bosons necessary to cool to $T=0$ :

$$
N_{b, 0}>\Delta N_{b}=\frac{C_{f, 0}}{k_{B} f(\eta)}\left[1+\frac{1}{\delta+3 / 2} \frac{C_{b, 0}}{C_{f, 0}}\right],
$$

where $T_{0}$ is the initial temperature, $C_{0}$ are the initial heat capacities and $\Delta N_{b}$ is the number of bosons evaporated. As the coolant is assumed to be initially a Bose gas with a condensate present, the initial number of bosons must exceed the saturated value of the population of the excited states:

$$
N_{b, 0}>A_{b} \Gamma(\delta+3 / 2) \zeta(\delta+3 / 2)\left(k_{B} T_{0}\right)^{\delta+3 / 2} .
$$

Note that, as an arbitrarily large number of particles can be stored in the condensate, no connection can be established between the number of particles and the heat capacity of the coolant. Thus condition (13) cannot be considered as a constraint on $C_{b, 0} / C_{f, 0}$ [20].

Recall that $\delta T / T \ll 1$ was required to replace the finite differences in (6) by derivatives. When the heat capacity of the Bose gas is initially much smaller that that of the Fermi gas, one finds that this condition is satisfied for any value of $\eta$, including $\eta=0^{+}$where $f(0)$ is finite and strictly positive. In this case, Eq. (11) and its consequences apply also when one evaporates all the non-condensed atoms at each cooling cycle.

\section{COOLING BY A BOLTZMANN GAS}

A Boltzmann gas has a total energy

$$
E_{b}\left(N_{b}, T\right)=(\delta+3 / 2) N_{b} k_{B} T .
$$

Equation (7) then reduces to

$$
\frac{\delta T}{\delta N_{b}}=\frac{[f(\eta)-(\delta+3 / 2)] T}{2 \alpha N_{f} \frac{T}{T_{F}}+(\delta+3 / 2) N_{b}} .
$$

The explicit dependence of the energy on $N_{b}$ introduces a negative contribution in the numerator. However, the total numerator remains positive, since one can show, by integration by parts, that the condition

$$
f(\eta)>\delta+3 / 2
$$

is satisfied for all $\eta>0$.

One finds that, by making a change of variables

$$
x \equiv \frac{2 \alpha}{\delta+3 / 2} \frac{T}{T_{F}} \frac{N_{f}}{N_{b}}=\frac{C_{f}}{C_{b}},
$$

the equation becomes separable. This leads to the constant of motion

$$
K=-\frac{\lambda}{\lambda-1} \ln |\lambda-1-x|+\frac{1}{\lambda-1} \ln x-\ln \left(N_{b} / N_{f}\right),
$$

where

$$
\lambda \equiv \frac{f(\eta)-(\delta+3 / 2)}{\delta+3 / 2}>0
$$

The asymptotic behavior of the temperature in the limit of the complete evaporation of the Boltzmann particles, i.e., $N_{b} \rightarrow 0$, falls into two regimes. The first is given by $x_{0}>\lambda-1$, where $x_{0}$ is the initial value of $x$. In this case, the temperature tends to a finite non-zero value due to an insufficient number of Boltzmann particles. The ratio of the final to initial temperature is

$$
\frac{T_{\text {final }}}{T_{0}}=\left[1-(\lambda-1) \frac{C_{b, 0}}{C_{f, 0}}\right]^{\lambda /(\lambda-1)} .
$$

The second regime is given by $x_{0}<\lambda-1$, which implies $\lambda>1$. The inequality $x_{0}<\lambda-1$ is equivalent to the requirement that there be a minimal initial number of Boltzmann particles:

$$
N_{b, 0}>\frac{C_{f, 0}}{k_{B}[f(\eta)-2(\delta+3 / 2)]} .
$$




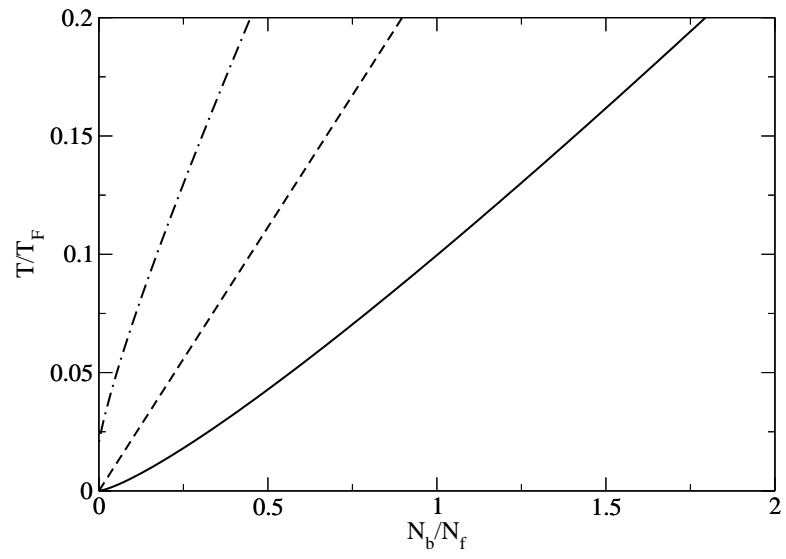

FIG. 3: Shown is the temperature of a degenerate Fermi gas subject to sympathetic cooling by a Boltzmann gas as a function of the number of Boltzmann particles. Three cases are depicted: $C_{f, 0} / C_{b, 0}=(\lambda-1) / 2$ (solid curve); $C_{f, 0} / C_{b, 0}=\lambda-1$ (dashed curve); and $C_{f, 0} / C_{b, 0}=2(\lambda-1)$ (dot-dashed curve). In the latter case the number of Boltzmann particles is insufficient to cool to $T=0$. Here the choice of parameters was $\eta=6$ for the energy cut-off parameter, $T_{0} / T_{F}=0.2$ for the initial temperature, and a harmonic trapping potential.

Note that $\lambda>1$ implies that the denominator is positive. Equation (22) may be equally expressed as a requirement on the initial heat capacities of the fermions and Boltzmann particles:

$$
C_{f, 0}<C_{b, 0}(\lambda-1) .
$$

In this case the temperature tends to zero as

$$
\frac{T}{T_{F}} \propto\left(\frac{N_{b}}{N_{f}}\right)^{\lambda} .
$$

In Fig. [3are shown three sample trajectories of Eq. (16). For $x_{0}>\lambda-1$ the final temperature is non-zero when all of the Boltzmann particles have been evaporated. For $x_{0}<\lambda-1$ the final temperature is zero and all of the Boltzmann particles are used in the evaporation. A third curve shows the case $x_{0}=\lambda-1$, which divides the two regimes: this corresponds to constant $x$, which implies $T / T_{F} \propto N_{b} / N_{f}$.
Note that, for a harmonic potential, $f(\eta)$ can be calculated exactly for a Boltzmann gas 18]. The critical value of $\eta$ which corresponds to $\lambda-1=0$ is then $\eta_{c}=4.59 \ldots$ For $\eta<\eta_{c}$ the final temperature of the fermions after evaporation of all the Boltzmann particles is always nonzero.

\section{CONCLUSION}

We have demonstrated that, in an idealized experiment with no particle losses besides those due to evaporation of the coolant, sympathetic cooling of degenerate Fermi gases is not limited by the heat capacity of the coolant when the latter is a Bose gas with a condensate present. In fact, it is advantageous to obtain $C_{f} / C_{b} \gg 1$ in order to minimize the number of bosons that must be evaporated, where $C_{f, b}$ are the heat capacities of the fermions and bosons. In this case, $T=0$ may be obtained provided that the initial number of bosons is sufficiently large:

$$
N_{b, 0}>2.7 N_{f} T_{0} / T_{F}
$$

where $N_{f}$ is the number of fermions, $T_{0}$ is the initial temperature which is much less than the Fermi temperature $T_{F}$, and $\eta=6$, a typical value of the energy cut-off for evaporative cooling, was chosen.

In contrast, in the case of a Boltzmann coolant, the final temperature of the fermions depends on the ratio of the initial values of the heat capacities $C_{f, 0} / C_{b, 0}$. For typical values of the evaporation cut-off parameter $\eta$, $C_{f, 0} / C_{b, 0}$ must be smaller than a critical value on the order of unity to reach $T=0$ after evaporation of all the Boltzmann particles.

Acknowledgments: We would like to thank Thomas Bourdel and Jean Dalibard for useful discussions, and Zoran Hadzibabic and Roland Combescot for useful comments on the manuscript. This work was supported by NSF grant no. mps-drf 0104447 Laboratoire Kastler Brossel is a research unit of l'Ecole Normale Supérieure and of l'Université Pierre et Marie Curie, associated with CNRS. We acknowledge financial support from Région Ile de France.
[*] Present address: JILA, National Institute of Standards and Technology and Physics Department, University of Colorado, Boulder, CO 80309-0440

[1] A. G. Truscott, K. E. Strecker, W. I. McAlexander, G. Partridge, and R. G. Hulet, Science 291, 2570 (2001).

[2] F. Schreck, L. Khaykovich, K. L. Corwin, G. Ferrari, T. Bourdel, J. Cubizolles, and C. Salomon, Phys. Rev. Lett. 87, 080403 (2001).

[3] G. Roati, F. Riboli, G. Modugno, and M. Inguscio, Phys. Rev. Lett. 89, 150403 (2002).
[4] Z. Hadzibabic, C. A. Stan, K. Dieckmann, S. Gupta, M. W. Zwierlein, A. Görlitz, and W. Ketterle, Phys. Rev. Lett. 88, 160401 (2002).

[5] Z. Hadzibabic, S. Gupta, C. A. Stan, C. H. Schunck, M.W. Zwierlein, K. Dieckmann, and W. Ketterle, Phys. Rev. Lett. 91, 160401 (2003).

[6] R. Onofrio and C. Presilla, Phys. Rev. Lett. 89, 100401 (2002).

[7] C. Presilla and R. Onofrio, Phys. Rev. Lett. 90, 030404 (2003). 
[8] K. Huang, Statistical Mechanics, 2nd ed. (John Wiley \& Sons, New York, NY, 1987).

[9] It is assumed for simplicity that the heat capacities are constant in the range of temperatures considered.

[10] H. F. Hess, Phys. Rev. B 343476 (1986).

[11] O. J. Luiten, M. W. Reynolds, and J. T. M. Walraven, Phys. Rev. A 53381 (1996).

[12] K.B. Davis, M.O. Mewes, W. Ketterle, Appl. Phys. B 60, 155 (1995).

[13] M. Wouters, J. Tempere, and J. T. Devreese, Phys. Rev. A 66043414 (2002).

[14] A gas of identical particles is in fact described by either Bose or Fermi statistics as the temperature approaches zero. However, for the sake of highlighting the effect of Bose statistics in contrast to classical statistics in the coolant, and in order to explain the meaning of the classical thermodynamic argument mentioned above, we shall imagine that Boltzmann statistics can be applied down to zero temperature.

[15] We do not claim here that zero temperature can be achieved in a real experiment: it would require an infinite time, as the collision rate between the fermions and the coolant tends to zero for $T \rightarrow 0$. Furthermore the semi- classical approximation used in this paper fails when the temperature drops below the energy of the first excited state of a particle of the coolant.

[16] C. Cohen-Tannoudji, Cours de physique atomique et moléculaire, http://www.lkb.ens.fr/ cct 1997.

[17] Note that such a density of states corresponds [16] to a trapping potential of form $V(x, y, z)=a|x|^{1 / \delta_{x}}+$ $b|y|^{1 / \delta_{y}}+c|z|^{1 / \delta_{z}}$ where $\delta_{x}+\delta_{y}+\delta_{z}=\delta$.

[18] In the case of harmonic trapping, $\delta=3 / 2$ is half integer so that $f(\eta)$ has a rather simple expression. For a Bose gas below $T_{c}$ we find $f(\eta)=\left[\eta^{3} g_{1}\left(e^{-\eta}\right)+3 \eta^{2} g_{2}\left(e^{-\eta}\right)+\right.$ $\left.6 \eta g_{3}\left(e^{-\eta}\right)+6 g_{4}\left(e^{-\eta}\right)\right] /\left[\eta^{2} g_{1}\left(e^{-\eta}\right)+2 \eta g_{2}\left(e^{-\eta}\right)+2 g_{3}\left(e^{-\eta}\right)\right]$ where the Bose function is $g_{\alpha}(z)=\sum_{k \geq 1} z^{k} / k^{\alpha}$. For a Boltzmann gas $f(\eta)=\left[\eta^{3}+3 \eta^{2}+6 \eta+6\right] /\left[\eta^{2}+2 \eta+2\right]$.

[19] Handbook of Mathematical Functions, edited by M. Abramowitz and I. A. Stegun (National Bureau of Standards, Washington, D. C., 1964).

[20] One may wonder if the condition (14) automatically implies (13). It turns out to be the case only if $\eta$ is large enough, that is if $(\delta+5 / 2) C_{f, 0}<\{[f(\eta) / f(0)]-[(\delta+$ $5 / 2) /(\delta+3 / 2)]\} C_{b, 0}$. 